\title{
ERVA-BALEEIRA: UMA POSSIBILIDADE REAL DA SOCIOBIODIVERSIDADE PARA MODELOS SUSTENTÁVEIS DE PRODUÇÃO
}

\author{
B. R. HARTWIG ${ }^{1}$, D. S. RODRIGUES ${ }^{2}$, C. J. F. DE OLIVEIRA JR ${ }^{3}$ \\ Universidade Federal de São Paulo ${ }^{1}$, Instituto de Botânica ${ }^{2,3}$ \\ floraacao@gmail.com ${ }^{3}$
}

Submetido 14/01/2020 - Aceito 17/04/2020

DOI: $10.15628 /$ holos.2020.9409

\begin{abstract}
RESUMO
A Biodiversidade brasileira é de extrema riqueza e alto potencial para uso econômico, incluindo espécies medicinais. É também uma importante estratégia para o desenvolvimento rural sustentável. Os estudos sobre fitotecnia e produção envolvendo as plantas nativas ainda são escassos perante o universo de possibilidades. O objetivo deste trabalho foi realizar uma revisão da cadeia produtiva da erva-baleeira (Cordia curassavica), como modelo de medicamento fitoterápico originado na flora nativa. A pesquisa foi realizada por meio de revisão da literatura científica, e as publicações foram analisadas e agrupadas de acordo com sua especificidade: botânica, ecologia, etnobotânica, fitoquímica e fitotecnia. Constatou-se um amplo uso popular da espécie, muitas
\end{abstract}

vezes associada à ação anti-inflamatória, creditada ao $\alpha$ humuleno, encontrado em cerca de $3 \%$ do óleo essencial. Outros componentes majoritários do óleo são $\alpha$-pineno, trans-cariofileno e alo-aromadendreno. A erva-baleeira é uma espécie nativa de uso medicinal que apresenta cadeia de produção em avançado estado de estruturação quando comparada a outras da flora brasileira. $\mathrm{Na}$ literatura científica, existem muitas informações acerca de sua ecologia, aspectos botânicos, uso por populações tradicionais e estudos fitoquímicos, porém são mais raros os estudos pertinentes à produção, assim, formas de manejo ainda ocorrem por extrativismo. Concluímos que há necessidade de investimentos em pesquisa $e$ produção de espécies medicinais.

PALAVRAS-CHAVE: Agroecologia, Uso econômico da Biodiversidade, Plantas medicinais, Cordia curassavica.

\section{BLACK SAGE: A REAL POSSIBILITY OF SOCIOBIODIVERSITY FOR SUSTAINABLE PRODUCTION MODELS}

\begin{abstract}
Brazilian biodiversity is extremely rich and has high potential for economic use, including medicinal species. It is also an important strategy for sustainable rural development. Studies on phytotechnics and production involving native plants are still scarce before the universe of possibilities. The aim of this study was to review the production chain of black sage (Cordia curassavica) as a model of herbal medicine originated in native flora. The research was conducted by reviewing the scientific literature, the publications were analyzed and grouped according to their specificity: botany, ecology, ethnobotany, phytochemistry and phytotechnics. We found widespread popular use of the species, often associated with anti-inflammatory action, credited to alpha-humulene, found in about $3 \%$ of the essential oil.
\end{abstract}

Other major components of the oil are alpha-pinene trans-caryophyllene and allo-aromadendrene. The blak sage is a native species of medicinal use that presents production chain in advanced state of structure when compared to others of the Brazilian flora. In the scientific literature there is a lot of information about its ecology, botanical aspects, use by traditional populations and phytochemical studies, but there are few studies related to production and management, so forms of management still occur through extraction. We concluded the need for investments in research and production of medicinal species.

KEYWORDS: Agroecology, Economic use of Biodiversity, Medicinal Plants, Cordia curassavica. 


\section{INTRODUÇÃO}

A biodiversidade nos biomas brasileiros é de extraordinária riqueza e representa uma infinidade de recursos e possibilidades de usos para a humanidade, sobretudo quando falamos de espécies medicinais (Funari; Ferro, 2005; Barreiro; Bolzani, 2009; Fernandes et al., 2013; Ribeiro et al., 2019). As plantas medicinais têm um longo e amplo histórico de uso por populações tradicionais e comunidades locais, sendo utilizadas sobretudo naquelas onde o serviço público de saúde é precário, ou mesmo em determinados contextos sociais, nos quais a baixa renda é um fator limitante ao acesso aos medicamentos industriais (Ribeiro et al., 2019). Esse conhecimento tradicional é comumente utilizado por universidades, institutos de pesquisa e indústrias farmacêuticas na prospecção de novos compostos para uso medicinal (Yunes; Calixto, 2001).

A bioprospecção pode ser definida como a busca de elementos da natureza, com vistas a seu uso e geração de produtos e processos na construção de cadeias de valor (Berlinck, 2012). A atividade de prospecção no Brasil em instituições de pesquisa data ainda do século XIX, tendo a exploração da pilocarpina, extraída da espécie Pilocarpus microphilus, como o primeiro programa de produção em maior escala (Berlinck, 2012).

As pesquisas com espécies medicinais, além do potencial de geração de novos produtos da sociobiodiversidade (Oliveira Jr. et al., 2018), entre eles medicamentos, promovem também o avanço de pesquisa básica e tecnológica, de caráter multidisciplinar, gerando possibilidades para o desenvolvimento sustentável no país (Barreiro; Bolzani, 2009). No oriente, a tradicional e milenar farmacopeia chinesa já tem possibilitado grandes avanços na farmacognosia, promovendo o surgimento de novos produtos para indústria farmacêutica (Barreiro; Bolzani, 2009).

No entanto, embora de alta potencialidade, a inovação tecnológica com a exploração de produtos da sociobiodiversidade no Brasil tem atingido apenas pequena escala de suas possibilidades. Por outro lado, grandes empresas de países altamente industrializados têm avançado no uso econômico de produtos derivados de plantas medicinais, muitas delas nativas da América do Sul (Funari; Ferro, 2005). Nesse cenário, é, ainda, relevante considerar o direito à repartição de benefícios econômicos originados no conhecimento tradicional (Azevedo, 2005; Funari; Ferro, 2005; Oliveira Júnior et al., 2012), muitas vezes negligenciado.

A sociobiodiversidade (Leonel, 2000) pode ser entendida como agregação de valor cultural à biodiversidade e tem sido apontada como um caminho promissor no uso econômico dos recursos naturais (Oliveira Júnior et al., 2018) para geração de renda e desenvolvimento local. Desse modo, espécies da flora nativa podem compor planos de manejo em agroecossistemas de maior equilíbrio ecológico, como os modelos agroecológicos e sistemas agroflorestais (Altieri, 1999; Amorozo, 2008; Haines-Young, 2009), com possibilidades, ainda, de incluir a prestação de serviços ambientais (Oliveira Júnior et al., 2014) e sequestro de carbono.

Os estudos sobre fitotecnia e a produção de matéria-prima envolvendo as plantas medicinais nativas ainda são escassos na literatura científica. Grande parte das plantas medicinais nativas comercializadas é ainda obtida por extrativismo (Fernandes et al., 2013). Pode-se 
considerar, ademais, que a atividade extrativista, com o aumento da demanda por matéria-prima pelo mercado globalizado, consequentemente, afeta as dinâmicas sociais das comunidades tradicionais, promovendo a superexploração dos recursos naturais (Fernandes et al., 2013), conforme descrito no trabalho clássico de Hardin (1968) "tragédia dos comuns". Ou seja, numa comunidade que tem determinado recurso que apresenta grande demanda, quando este se esgota, busca-se por novas áreas, num processo cíclico de exploração e degradação ambiental (Hardin, 1968; Fernandes et al., 2013).

A minimização dos problemas causados pela superexploração ou extrativismo irracional é ainda um desafio, pois o estudo para estabelecimento de novas cadeias produtivas a partir da utilização de espécies medicinais nativas é bastante complexo e de caráter interdisciplinar, pois envolve a etnobotânica, o conhecimento popular, o conhecimento no manejo, produção ou extrativismo, estudos químicos, bioquímicos e farmacológicos, processos de beneficiamento e logísticas de mercado (Maciel et al., 2002).

Diante disso, o objetivo deste trabalho foi realizar uma revisão sobre o conhecimento nas diversas áreas do conhecimento da cadeia produtiva da erva-baleeira (Cordia curassavica (Jacq.) Roem \& Schult), utilizada como modelo de medicamento fitoterápico originado na flora nativa na promoção do desenvolvimento sustentável.

\section{METODOLOGIA}

O estudo foi realizado por meio de revisão bibliográfica. Foram consultadas diferentes bases de dados, como Portal de Periódicos da CAPES, Scientific Electronic Library Online (SciELO), Web of Science ISI e Google Scholar. As palavras-chave utilizadas foram: erva-baleeira, Cordia curassavica, Varronia curassavica e Cordia verbenacea. Os estudos que continham informações sobre botânica, aspectos ecológicos, etnobotânicos, fitoquímicos e aspectos fitotécnicos da espécie foram selecionados para a presente revisão, e outras referências não encontradas na busca, porém encontradas nas referências dos trabalhos selecionados, foram também incluídas. Foram selecionados artigos, teses e dissertações concernentes ao tema para a coleta de dados e análise conforme o objetivo deste estudo, facilitando o entendimento do estado atual do conhecimento sobre a cadeia produtiva da erva-baleeira.

Com o intuito de aprofundar a análise dos trabalhos coletados e permitir uma visão mais abrangente do estado atual da cadeia de produção, os tópicos foram divididos em: 1) Botânica; 2) Ecologia; 3) Etnobotânica; 4) Fitoquímica; 5) Fitotecnia; 6) Políticas Públicas, Regulamentações e Mercado. Para cada tópico, foram analisados diferentes estudos apresentados de forma quantitativa na Tabela 1, considerando que cada referência pode abordar mais de um assunto para estudo e análise. 
Tabela 1 - Referências utilizadas para a revisão bibliográfica sobre a erva-baleeira (Cordia curassavica (Jacq.) Roem \& Schult)

\begin{tabular}{c|c}
\hline Assuntos & Número de Referências \\
\hline Botânica & 28 \\
Ecologia & 20 \\
Etnobotânica & 18 \\
Fitoquímica & 34 \\
Fitotecnia e mercado & 12 \\
Outros temas & 13 \\
\hline
\end{tabular}

\section{RESULTADOS E DISCUSSÃO}

\subsection{Botânica}

Devido à ampla distribuição geográfica da erva-baleeira e de seu uso terapêutico pela medicina popular, diversos outros nomes são também utilizados para ela: camarinha, camarona, balieira-cambará, erva-preta, catinga-preta, salicina, pimenteira, catinga-de-barão, mariamilagrosa, maria-preta, camaroneira-do-brejo. Porém, o nome mais utilizado é mesmo ervabaleeira, o qual é apontado como sendo dado por nativos de Santa Catarina, que atuavam como caçadores de baleia e utilizavam a espécie para cura de ferimentos relacionados à atividade (Lorenzi; Matos, 2008). Em inglês, a espécie é chamada de black sage ou maggy-plant devido ao seu odor característico, semelhante a temperos prontos para carnes (Montanari Junior, 2000).

A espécie pertence à família Boraginaceae, que possui cerca de 100 diferentes gêneros e mais de duas mil espécies, sendo subdividida em: Ehretiodeae, Cordiodeae, Helitropioideae e Boraginoideae (Miller; Gottschling, 2007; Falkenberg, 2011). De acordo com Ficarra et al. (1995), o centro de diversidade do gênero Cordia está situado na zona tropical da América e nas regiões tropicais e subtropicais da África, Austrália e Ásia.

A classificação da erva-baleeira é um assunto bastante discutido e controverso. De acordo com Miller e Gottschling (2007), as incertezas ocorrem devido à ampla variação morfológica das espécies que constituem o gênero Cordia, o qual possui cerca de 250 espécies e é dividido nos subgêneros: Myxa, Varronia e Cordia. Porém, novos estudos moleculares propõem que estes subgêneros sejam elevados à condição de gênero, devido a diferenças morfológicas detectadas (Miller; Gottschling, 2007). Gasparino e Barros (2009) mostram que a diferenciação entre estes gêneros é dada especificamente pela morfologia polínica: enquanto o subgênero Varronia possui grãos de pólen 3-porados, Cordia possui grãos de pólen 3-colporados.

O nome aceito de acordo com o The Plant List (2013) e IPNI (2012) é Cordia curassavica (Jacq.) Roem \& Schult; pertencente ao gênero Cordia e família Boraginaceae. Já de acordo com a Flora do Brasil (2010), o nome aceito é Varronia curassavica Jacq., sendo C. verbenacea e $C$. curassavica seus sinônimos. As sinonímias mais encontradas para a espécie são: Varronia curassavica (Jacq) e Cordia verbenacea (DC). Porém, além destas, outras são registradas em diferentes literaturas: C. salicina DC, C. cylindristachia Auctt. Bras. ex Fresen, Lithocardium fresenii Kuntze, L. salicinum Kuntze, e L. verbaceum Kuntze (Sánchez, 1995). 
A erva-baleeira (Figura 1) é uma espécie arbustiva com ramos flexíveis e altura entre 2 e 3 metros, de hábito perene (Ribeiro; Diniz, 2008). Suas folhas são extremamente aromáticas e podem atingir em média 10 centímetros de comprimento, são hipoestomáticas, simétricas e apresentam ápice agudo e base decorrente, lâmina lanceolada com borda denteada (Leal-Costa; Amélia, 2017). A disposição das folhas ocorre de forma alternada, raramente oposta, e a coloração verde da face adaxial é mais escura do que a face abaxial (Lorenzi; Matos 2008). Suas inflorescências são racemosas, formando-se em espigas curvadas para baixo com até 15 centímetros de comprimento com pequenas flores brancas, geralmente pentâmeras radiais e diclamídeas (Gilbert; Favoreto, 2012; Hoeltgebaum et al., 2018). De acordo com Hoeltgebaum et al. (2018), as flores da erva-baleeira possuem curta duração, a abertura dos botões florais ocorre durante o dia e a murcha começa a ocorrer cerca de oito horas após a antese, aumentando gradativamente sua oxidação. Apesar da murcha da corola, as estruturas reprodutivas e o estigma continuam ativos até a manhã seguinte, com o início de um novo ciclo reprodutivo. Sua simetria floral é zigomorfa, possui corola gamopétala bilabiada com 4 a 7 milímetros de comprimento. 0 cálice é gamossépalo, lobado, campanulado e verde. A espécie é hermafrodita com ovário do tipo súpero bilocular e bicarpelar com dois óvulos em cada lóculo, estilete ginoblástico e androceu composto por 5 estames alternados com os lobos da corola (Smith, 1970; Hoeltgebaum et al., 2018). Seus frutos são drupáceos, subglobosos, cariopses, esféricos e vermelhos quando maduros (Smith, 1970; Silva Jr et al., 1995; Hoeltgebaum et al., 2018), com aproximadamente 0,4 centímetros, distribuindo-se em diferentes alturas dentro do mesmo eixo principal.

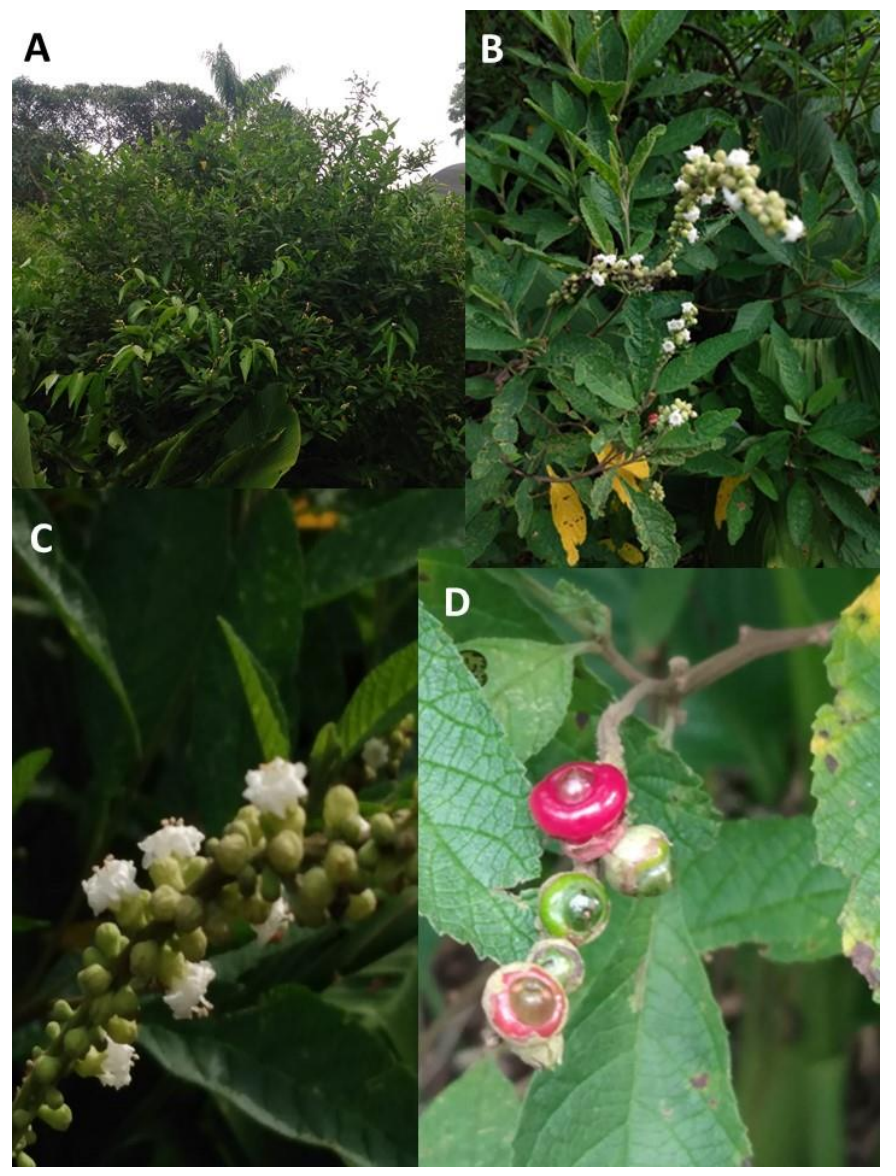

Figura 1. Aspectos morfológicos da erva-baleeira (Cordia curassavica). A) geral, B) inflorescência, C) flor, D) frutos. (Fotos: Oliveira Jr., C.J.F.) 


\subsection{Ecologia}

A erva-baleeira é uma espécie heliófita nativa da Mata Atlântica, com ampla distribuição pelo litoral brasileiro (Fernandes et al., 2007), variando em altitude desde o nível do mar até altas montanhas (Sánchez, 1995). Exemplares foram identificados também na região Amazônica e nos pampas do Rio Grande do Sul (Carvalho Júnior et al., 2004), no interior dos estados de São Paulo, Minas Gerais e Goiás (Montanari Junior, 2011) e na caatinga de Pernambuco, em regiões de florestas de altitude e regiões mais baixas e secas com predominância de vegetação xerófita (Rodal; Nascimento, 2002).

O florescimento é mais intenso durante as estações da primavera e verão, quando ocorre o aumento da temperatura e umidade (Montanari Junior, 2000). Na região da caatinga, Melo e Sales (2005) apresentaram estudo em que a floração das plantas ocorreu durante todo o ano, porém mais intensamente entre março e maio, meses tipicamente mais frios e secos.

A dispersão das sementes da erva-baleeira é zoocórica (Marques; Oliveira, 2005) e a sua polinização é entomófila (Smith, 1970; Montanari Junior, 2011). Seus frutos são fonte de alimento para pássaros de várias espécies, que acabam por dispersar suas sementes (Michielin et al., 2009; Hoeltgebaum et al., 2018). Suas flores são visitadas principalmente por abelhas, formigas e borboletas, e, de acordo com Moura et al. (2007), a erva-baleeira exerce uma forte influência sobre a abundância de abelhas na região do Rio São Francisco, por apresentar floração contínua, favorecendo a estabilidade das comunidades de seus dispersores.

A erva-baleeira libera o $\alpha$-pineno, que atrai fêmeas do coleóptero Cratosomus flavofasciatus (Curculionidae), que, por sua vez, liberam o feromônio frontalina, atraindo os machos até a planta, que passa, então, a servir como sítio de reprodução (Nascimento et al., 1986). A presença de formigas nos ramos da erva-baleeira é comum devido a nectários extraflorais, e, embora não reduza o número de polinizadores de rápida visitação, pode proteger outros insetos sugadores, como pulgões e cochonilhas. Existem relatos de visitas por insetos das ordens Coleoptera, Hemiptera, Diptera e Hymenoptera (Brandão et al., 2015; Hoeltgebaum et al., 2018).

\subsection{Etnobotânica}

Há registros do uso da erva-baleeira por comunidades tradicionais no tratamento de inflamações, dores musculares e da coluna, artrites, reumatismos, úlceras estomacais e também como tônico para contusões (Passos et al., 2007; Medeiros et al., 2007; Roldão et al., 2008; Gomes et al., 2010). Além disso, externamente, ela tem uso citado como cicatrizante em feridas e regiões inflamadas. Popularmente, o uso é feito a partir das folhas em forma de infusão, decocção, pomadas, tinturas, extratos hidroalcoólicos e cataplasmas (Blanco, 2013). Outros registros de seu uso por populações locais estão descritos em Melo et al. (2008), Gandolfo e Hanazaki (2011) e Falkenberg (2011).

No povoado de Sapucaia, localizado no Recôncavo Baiano, Rodrigues et al. (2006) apresentam um estudo etnobotânico no qual a erva-baleeira, citada como maria-milagrosa, é utilizada para amenizar tonturas. Nas comunidades da llha do Cardoso/SP e llha de Santa Catarina/SC, estudadas por Miranda e Hanazaki (2008), a erva-baleeira foi uma das espécies nativas medicinais de ampla citação, sendo apontada pelos moradores de todas as regiões. No litoral paulista, a pesquisa realizada por Araújo (2007) identificou a presença da espécie erva- 
baleeira, mas também com a variação fonética, erva-baliera, nas seguintes comunidades: Bonete, município de Ilhabela/SP; Barra do Una, município de Peruíbe/SP; e na Praia do Una, município de Iguape/SP, sendo as plantas identificadas tanto em quintais como em zonas de vegetação nativa. O uso tradicional da erva-baleeira na região da Praia Grande em Arraial do Cabo/RJ foi descrito por Fonseca-Kruel et al. (2004) como uma espécie usada pela comunidade de forma medicinal e alimentar, onde as folhas e frutos da caimbê-preto (erva-baleeira) são consumidos. No Vale do Paraíba/SP, Alvarenga et al. (2017) realizaram um levantamento etnobotânico de espécies comercializadas em mercados e feiras livres no qual o consumo do chá das folhas da erva-baleeira era indicado para o tratamento de diabetes.

O levantamento de dados sobre as plantas medicinais na cultura ítalo-brasileira no município de Riozinho, no Rio Grande do Sul (Koch, 2000), registra que a baleeira era usada popularmente em casos de diarreia e hemorroidas. Na comunidade do Pântano do Sul, localizada em Florianópolis/RS, Melo et al. (2008) apresentam estudo sobre espécies de restinga e explicitam que a erva-baleeira, ali conhecida como camarona, é, assim como no município de Riozinho/RS, utilizada no tratamento de hemorroidas. Seu chá também é usado contra reumatismo, dores de barriga e verminoses. A espécie também é citada em estudos realizados na Reserva Biológica de Poço das Antas/RJ (Christo et al., 2006), nas comunidades da Marambaia e Camboinhas (Itacaré, BA) (Pinto et al., 2006), no município de Cananéia/SP (Oler et al., 2019) e nas feiras livres da zona norte e sul do município do Rio de Janeiro/RJ (Maioli-Azevedo et al., 2007). Descrições internacionais do uso da planta mostram que ela é usada para tratamento de diarreia, no México, e controle de parasitas em animais domésticos em Trinidad-Tobago (Lans et al., 2000; Hernández et al., 2003).

\subsection{Fitoquímica}

De acordo com Scardelato et al. (2016), a erva-baleeira apresenta em sua composição óleos essenciais, lipídios (ácidos graxos, esteroides), compostos fenólicos (taninos, flavonoides), substâncias terpênicas, pectinas, saponinas e alcaloides. Os principais componentes do óleo essencial de erva-baleeira fazem parte dos grupos mono e sesquiterpenos (Carvalho Júnior et al., 2004; Santos et al., 2007; Brandão et al., 2017; Sciarrone et al., 2017; Alves et al. 2019) e são apresentados na Tabela 2. Outros compostos foram identificados em menores proporções, como $\alpha$-cedreno, $\alpha$-cis-bergamoteno, $\alpha$-(E,E)-farneseno, citronelol, $\beta$-elemeno, $\beta$-felandreno, $\beta$ gurjuneno, $\beta$-sinensal, (Z)- $\alpha$-trans-bergamotol, (Z)- $\beta$-farneseno, biciclogermacreno, espatulenol, (Z)-nerolidol, cadineno, carotol, epóxi-cariofileno e (Z)-y-bisaboleno (Carvalho Júnior et al., 2004; Alves et al., 2019; Queiroz et al. 2020). O estudo desenvolvido por Facanali et al. (2020) possibilitou a identificação de 135 constituintes do óleo essencial da erva-baleeira com uso da cromatografia gasosa bidimensional. 
Tabela 2 - Principais compostos químicos presentes no óleo essencial de erva-baleeira (Cordia curassavica (Jacq.) Roem \& Schult)

\begin{tabular}{|c|c|c|}
\hline Principais compostos ativos do óleo essencial & Proporção (\%) & Referência \\
\hline$\alpha$-pineno & $\begin{array}{l}29,69 \\
45,00 \\
24,48 \\
22,20\end{array}$ & $\begin{array}{c}\text { Carvalho Júnior et al. (2004) } \\
\text { Santos et al. (2007) } \\
\text { Sciarrone et al. (2017) } \\
\text { Alves et al. (2019) }\end{array}$ \\
\hline trans-cariofileno & $\begin{array}{c}25,27 \\
19,90 \\
7,40\end{array}$ & $\begin{array}{c}\text { Carvalho Júnior et al. (2004) } \\
\text { Santos et al. (2007) } \\
\text { Sciarrone et al. (2017) }\end{array}$ \\
\hline alo-aromadendreno & $\begin{array}{l}9,99 \\
5,00\end{array}$ & $\begin{array}{l}\text { Carvalho Júnior et al. (2004) } \\
\text { Santos et al. (2007) }\end{array}$ \\
\hline$\alpha$-humuleno & $\begin{array}{l}4,64 \\
3,90 \\
2,13\end{array}$ & $\begin{array}{c}\text { Carvalho Júnior et al. (2004) } \\
\text { Santos et al. (2007) } \\
\text { Sciarrone et al. (2017) }\end{array}$ \\
\hline$\alpha$-santaleno & $\begin{array}{l}17,30 \\
35,60\end{array}$ & $\begin{array}{c}\text { Sciarrone et al. (2017) } \\
\text { Alves et al. (2019) }\end{array}$ \\
\hline
\end{tabular}

As variações dos metabólitos secundários podem ser justificadas devido às variáveis ambientais como clima, intensidade e duração da radiação recebida, ação de predadores, altitude, estresse hídrico, tipo de solo, formas de manejo e idade foliar (Feijó et al., 2014), além do próprio método utilizado para extração e análise.

A secreção do óleo ocorre através de tricomas glandulares situados na superfície abaxial foliar (Leal-Costa; Amélia, 2017). De acordo com estudo realizado por Ventrella e Marinho (2008), existem duas classes de tricomas glandulares presentes na região internerval das folhas: globulares e reniformes. Os globulares secretam os terpenoides presentes no óleo essencial e os reniformes secretam os compostos fenólicos, incluindo os flavonoides. Já os tricomas não glandulares estão divididos em curtos e longos, e, ao contrário dos glandulares, distribuem-se de forma mais escassa ao longo da superfície foliar.

O $\alpha$-humuleno é um sesquiterpeno monocíclico considerado o principal marcador do óleo essencial da erva-baleeira. Ele atua bloqueando a enzima ciclo-oxigenase-2, que está diretamente relacionada com a produção da prostaglandina, atuante em processos de inflamação do organismo (Medeiros et al., 2007). Além do $\alpha$-humuleno, foi encontrado outro sesquiterpeno bicíclico chamado trans-cariofileno e encontradas duas estruturas triterpenicas com esqueleto de carbono do damarano. As estruturas foram nomeadas como cordialina A e cordialina B, classificadas como triterpenos tetracíclicos (Barroso et al., 2002).

Os estudos sobre os efeitos biológicos e bioquímicos dos compostos de erva-baleeira são apresentados na Tabela 3. 
Tabela 3: Efeitos terapêuticos e estudos farmacológicos da erva-baleeira (Cordia curassavica (Jacq.) Roem \& Schult)

\begin{tabular}{|c|c|}
\hline Efeito & Referência \\
\hline Analgésico & $\begin{array}{l}\text { Sertié et al. (2005); Pianowsky (2005); Roldão et } \\
\text { al. (2008) }\end{array}$ \\
\hline Anti-inflamatório & $\begin{array}{l}\text { Sertié et al. (1988, 1991, 2005); Bayeux et al. } \\
\text { (2002); Carvalho Júnior et al. (2004); Ticli et al. } \\
\text { (2005); Pianowsky (2005); Brandão et al. (2006); } \\
\text { Medeiros et al. (2007); Passos et al. (2007); } \\
\text { Fernandes et al. (2007); Parisotto et al. (2012) }\end{array}$ \\
\hline Antiulcerogênico & Sertié et al. (1991); Roldão et al. (2008) \\
\hline Antimicrobiano & $\begin{array}{c}\text { Hernándes et al. (2003); Carvalho Júnior et al. } \\
\text { (2004); Meccia et al. (2009); Matias et al. (2010); } \\
\text { Michielin et al. (2009); Rodrigues et al. (2012); } \\
\text { Pinho et al. (2012); Alves et al. (2014); Matias et } \\
\text { al. (2017) }\end{array}$ \\
\hline Antiofídico & Ticli et al. (2005) \\
\hline Antifúngico & $\begin{array}{l}\text { Carvalho Júnior et al. (2004); Rodrigues et al. } \\
\text { (2012); Barros et al. (2019) }\end{array}$ \\
\hline Anticancerígeno & Parisotto et al. (2012) \\
\hline Antialérgico & Fernandes et al. (2007) \\
\hline Antioxidante & Santos et al. (2004) \\
\hline Ação Relacionada Ao Aedes Aegypti & Santos et al. (2006) \\
\hline Ação Relacionada Ao Schistosoma Mansoni & Frezza et al. (2010) \\
\hline
\end{tabular}

\subsection{Fitotecnia}

A erva-baleeira apresenta rápido crescimento e não é exigente quanto ao solo. Seu crescimento pode ocorrer em áreas de clareira, com exposição direta ao sol, e também em condições de sombra parcial, sob outras espécies (Ribeiro; Diniz, 2008). Segundo estes autores, ela apresenta bom desenvolvimento em solos com pH entre 4 e 5,5. As condições de iluminação influenciam o desenvolvimento da planta e podem causar variações morfofisiológicas e na produção do óleo essencial.

A propagação pode ser feita por sementes ou estacas. A melhor época para plantio é no início do período das chuvas, e a primeira colheita já pode ser realizada após um ano (Ribeiro; Diniz, 2008; Blanco, 2013). Para produção de mudas via sementes, é necessário coletar os frutos maduros, extrair as sementes e colocá-las para germinar entre 40 e 50 dias. A taxa de germinação da erva baleeira é em média de 78\% (Figueira et al., 2001). Machado et al. (2011) estudaram o comportamento fisiológico das sementes da erva-baleeira variando a luz, o substrato utilizado e a temperatura e, de acordo com os resultados encontrados, recomendam que a melhor temperatura para germinação é entre $15^{\circ} \mathrm{C}$ e $20^{\circ} \mathrm{C}$. É interessante considerar que a propagação realizada por sementes forma indivíduos com maior variação genética, o que pode não ser interessante para o objetivo de produção comercial do óleo essencial, mas é fundamental para a conservação e 
preservação da base genética da espécie, podendo servir para o desenvolvimento de novas variedades, mais produtivas ou com resistência a fatores adversos.

A propagação com uso de estacas é uma técnica viável comercialmente e permite a reprodução de genótipos de interesse (Fachinello et al., 2005). Suas estacas são semi-lenhosas e, de acordo com Mendes et al. (2014), 52,5\% alcançam o enraizamento. Para potencializar o enraizamento da erva-baleeira, Rodrigues et al. (2010) testaram a aplicação de extrato de tiririca em diferentes concentrações, porém não encontraram diferenças no enraizamento das estacas nas condições estudadas. Um estudo semelhante, realizado por Caye et al. (2020), comparou o efeito do uso de fitohormônios e extrato de tiririca na indução de raízes em estacas e obteve melhores resultados com o uso do hormônio industrial ácido indolbutírico (AIB).

Existem poucos relatos sobre modelos de produção da erva-baleeira, bem como dados sobre produção e produtividade. Os poucos sistemas existentes de plantio da erva-baleeira são, em geral, monoculturas, e, como consequência, tornam-se mais susceptíveis às pragas e doenças. Rosa et al. (2008) registraram ataque de Dictyla monotropidia Stal em espécimes de erva-baleeira numa plantação localizada no município de Jaú/SP.

Devido ao princípio ativo e aos óleos essenciais da erva-baleeira serem retirados das folhas, grande parte da literatura encontrada está relacionada a formas de potencializar a produção da fração aérea da planta. De acordo com Morandi (2009), uma área de cultura da erva-baleeira com genótipos selecionados e plantas com mais de três anos pode chegar a produzir 16 mil quilos de biomassa por hectare em um ano. Essa quantidade de biomassa pode atingir a produção de 20 litros de óleo essencial por hectare/ano, considerando que o valor comercial do óleo essencial da erva-baleeira pode chegar a 16 mil reais/litro (Oshadhi, 2020).

Brandão et al. (2017) estudaram a produção de folhas (massa fresca - $\mathrm{kg} / \mathrm{ha}$ ) e óleos essenciais e encontraram que espaçamentos mais adensados $(1,0 \times 0,5 \mathrm{~m})$ com cobertura morta (mulch) proporcionaram os melhores rendimentos de massa foliar. Silva Jr et al. (1995) realizaram um estudo que associava a resposta da erva-baleeira mediante a introdução de diferentes doses de nutrientes e identificaram que, em solos com baixa fertilidade, a produção de folhas era reduzida. Esta informação condiz com estudos de Montanari Jr. (2000), segundo os quais a produção de artemetina e hidroxi-artemetina aumentou após a incorporação de nitrogênio, fósforo e potássio no solo.

O método de extração de óleos essenciais mais utilizado é o processo de arraste a vapor em aparelho tipo Clevenger. Os vapores resultantes do aquecimento contêm as substâncias voláteis, que posteriormente são condensadas, e, por fim, o óleo essencial é separado da fase aquosa por diferença de densidade (Rodrigues et al., 2011). Rodrigues et al. (2012) obtiveram $0,47 \%$ de rendimento do óleo essencial com uso de folhas frescas em base seca e $1,92 \%$ de rendimento com uso de folhas secas também em base seca. No experimento realizado por Passos et al. (2007), o método utilizado foi o mesmo (arraste a vapor com aparelho Clevenger), porém o rendimento das folhas frescas foi de $0,37 \%$. O padrão de qualidade ideal para a venda na indústria farmacêutica é que o óleo essencial contenha o teor mínimo de $2 \%(v / v)$ de $\alpha$-humuleno (Brandão et al., 2017). 


\subsection{Políticas públicas, regulamentações e mercado}

A erva-baleeira possui grande importância econômica e científica devido ao uso do seu óleo essencial na produção do primeiro medicamento fitoterápico desenvolvido no Brasil e originado na flora nativa, chamado Acheflan ${ }^{\circledR}$. Seu uso é indicado no tratamento de tendinite crônica e dores miofasciais devido ao composto ativo $\alpha$-humuleno, o qual possui atividade anti-inflamatória (Quispe-Condori et al., 2008). O medicamento possui, em sua composição, 2,3 a 2,9\% de $\alpha$ humuleno e $\beta$-cariofileno como princípios ativos (Quispe-Condori et al., 2008), sendo comercializado como aerossol ou pomada. Atualmente, o produto é comercializado em 13 países, incluindo o Brasil, Canadá, Chile, Estados Unidos, Japão, Peru, e pretende expandir seu mercado englobando o México. O processo de aprovação da comercialização internacional foi realizado pela Food and Drug Administration (FDA) (Bolzani et al., 2012). No Brasil, a aprovação da comercialização foi feita pela ANVISA (Agência Nacional de Vigilância e Saúde) no ano de 2004 (Gilbert; Favoreto, 2012). Além do Acheflan ${ }^{\circledR}$, existe, no mercado farmacêutico, o fitoterápico Cordia Extreme ${ }^{\mathrm{TM}}$, produzido pela empresa americana PhytoRich.

O desenvolvimento do fitomedicamento causou um grande impacto no cenário da indústria farmacêutica brasileira devido à espécie ser amplamente usada por comunidades tradicionais, demonstrando a grande importância do conhecimento popular e do incentivo às pesquisas etnobotânicas. Este conhecimento popular é protegido por leis brasileiras (Azevedo, 2005) e seu uso econômico deve reverter benefícios econômicos para as comunidades nas quais o conhecimento foi acessado. No entanto, neste caso, por ser um conhecimento bastante difuso, ele dificulta a identificação de sua origem e, consequentemente, o acesso das políticas sobre repartição de benefícios (Oliveira Júnior et al., 2012). Em casos como este, Oliveira Júnior et al. (2012) sugerem a criação de um fundo rotatório para que se possa financiar iniciativas junto às comunidades tradicionais detentoras de conhecimento tradicional.

O setor produtivo dessa espécie também cresceu (Lourenzani, 2004), porém de forma bastante desorganizada, visto que o extrativismo das plantas ainda ocorre em remanescentes de áreas naturais. Esse tipo de manuseio desenfreado e sem estratégia acarreta diversos danos em escala ecossistêmica, podendo resultar em extinções locais, conforme descrito na teoria "tragédia dos comuns" (Hardin, 1968).

O Brasil possui algumas políticas e instrumentos norteadores do desenvolvimento de ações para incentivo ao uso de plantas medicinais na saúde pública. Entre eles está a Política Nacional de Plantas Medicinais e Fitoterápicos (PNPMF), aprovada em 2006, e a Política Nacional de Práticas Integrativas e Complementares (PNPIC), instaurada no Sistema Único de Saúde (SUS), que, atualmente, possui uma lista com 71 espécies de plantas medicinais, da qual a erva-baleeira faz parte (Brasil, 2006). A Secretaria Estadual da Saúde em Minas Gerais lançou o Programa Componente Verde da Rede de Farmácia de Minas, em 2010, para incentivar o uso de fitoterápicos e facilitar o acesso às plantas medicinais através do SUS. Foram selecionadas 16 espécies de plantas e, entre elas, está a erva-baleeira.

A erva-baleeira pertence, também, ao Formulário Nacional Fitoterápico da Farmacopeia Brasileira como anti-inflamatório a partir do uso tópico de suas folhas, via infusão, compressa ou pomada. Com a implementação da PNPIC no SUS, a utilização de plantas medicinais foi legalizada e incentivada (Pereira; Albiero, 2015), visando também trazer mais garantia, segurança e eficácia, 
pois, mesmo sendo um produto natural, não está livre de efeitos colaterais e danos à saúde por uso inapropriado, ou mesmo de adulterações e falsificações no comércio.

De acordo com a pesquisa feita por Souza et al. (2012) sobre cadeia produtiva e comercialização de plantas medicinais em Minas Gerais, existe alta demanda pelas plantas medicinais, porém a oferta é precária e o produto vegetal costuma ter má qualidade, devido à carência de informação em diversos elos da cadeia de produção. Lourenzani et al. (2004) afirmam que a estrutura do mercado de fitoterápicos poderia ser baseada em diferentes vias de comercialização (informal, farmácias de manipulação e indústria), satisfazendo a demanda por meio do incentivo à produção local de plantas.

\section{CONSIDERAÇÕES FINAIS}

Muitos autores têm apontado o uso da biodiversidade nativa em sistemas de produção, aumentando a resiliência e a sustentabilidade dos agroecossistemas (Altieri, 1999; Oliveira Jr. et al. 2018). Assim, a espécie é indicada para a produção em modelos agroecológicos, como policultivos e sistemas agroflorestais, apresentando-se como excelente opção em programas de desenvolvimento rural sustentável (Oliveira Jr.; Cabreira, 2012). Pode ser, ainda, utilizada em processos de recuperação de áreas degradadas e em plantios legais passíveis de produção comercial em áreas de proteção, como Reservas Legais (RLs) e Áreas de Preservação Permanente (APPs). O cultivo desta espécie configura sua conservação on farm (Santonieri; Bustamonte, 2016), reduzindo, deste modo, o extrativismo e processos de superexploração em ambientes naturais, o que, consequentemente, promove sua preservação e conservação. A espécie presta importante serviço ambiental, servindo de hospedagem e alimentação para polinizadores e dispersores. Além do aspecto ecológico, é uma alternativa promissora para a geração de renda e trabalho no campo, sobretudo quando falamos da agricultura familiar, que, geralmente, apresenta produção mais diversificada.

A erva-baleeira é uma espécie nativa de uso medicinal que apresenta cadeia de produção em avançado estado de estruturação quando comparada a outras espécies da flora brasileira. $\mathrm{Na}$ literatura acadêmica, existem muitas informações acerca de sua ecologia, aspectos botânicos, uso por populações tradicionais e estudos clínicos, além dos aspectos relacionados à legislação de seu uso econômico, porém são mais raros os estudos pertinentes à produção e a formas de manejo. A obtenção de matéria-prima ainda ocorre em forma de extrativismo, colocando em risco os remanescentes e fragmentos florestais de mata nativa. Consideramos a importância de mais incentivos na pesquisa científica para produção da espécie. A construção de políticas públicas para o uso econômico da biodiversidade depende, além das pesquisas em produção, de mercados estruturados e de padrões de qualidade da matéria-prima para sua efetiva implantação.

\section{REFERÊNCIAS BIBLIOGRÁFICAS}

ALTIERI, M. A. (1999). The ecological role of biodiversity in agroecosystems. Agriculture, Ecosystems and Environment, 74: 19-31. https://doi.org/10.1016/S0167-8809(99)00028-6. 
ALVARENGA, C. F.; LIMA, K. M. N.; MOLLICA, L. R.; AZEREDO, L. O.; CARVALHO, C. (2017). Uso de plantas medicinais para o tratamento do diabetes mellitus no Vale do Paraíba-SP. Revista Ciências e Saúde, 2(2):36-44.

ALVES, E. F.; SANTOS, B. S.; MATIAS, E. F. F. (2014). Avaliação da atividade antibacteriana e modulatória da fração hexânica do extrato hexânico de Cordia verbenacea DC. Revista Interfaces: Saúde, Humanas e Tecnologia, 2(5). https://dx.doi.org/10.16891/2317.434X.96.

ALVES, M. S.; SILVA, L. C. P.; PEREIRA, E. A. D.; ESPÓSITO, E. P.; FAGUNDES, L. M.; FARIA, T. S.; SANTOS, A. M.; CHAVES, D. S. A.; CASTRO, R. N.; SOUZA, M. A. A. (2019). Diversidade química dos óleos essenciais de plantas do Jardim Botânico da UFRRJ e de outras Localidades, baseado em análise de imagem e estatística multivariada. Rev. Virtual Quim., 11 (5), 1635-1656. https://doi.org/10.21577/1984-6835.20190115.

AMOROZO, M. C. M. (2008). Os quintais: funções, importância e futuro. In: GUARIM NETO, G; CARNIELLO, M. A. (Orgs.). Quintais matogrossenses: espaços de conservação e reprodução de saberes. Cáceres: UNEMAT, p. 15-26.

ARAÚJO, L. G. (2007). Etnobotânica Caiçara: diversidade e conhecimento de recursos vegetais no litoral paulista. Dissertação (mestrado) - Universidade Estadual de Campinas, Instituto de Biologia, Campinas, SP. 210p.

AZEVEDO, C. M. A. (2005). A regulamentação do acesso aos recursos genéticos e aos conhecimentos tradicionais associados no Brasil. Biota Neotropica, 5(1): BN00105012005. http://dx.doi.org/10.1590/S1414-753X2011000100013.

BARREIRO, E. J.; BOLZANI, V. S. (2009). Biodiversidade: fonte potencial para descoberta de fármacos. Química Nova, 32(3): 679-688. http://dx.doi.org/10.1590/S0100$\underline{40422009000300012 .}$

BARROS, R. O.; BEZERRA, V. B.; LUCENA, A. L. V. M.; LEITE, V. D.; FEITOSA, J. H. F.; MANGUEIRA, C. E. A.; CALIXTO, C. G.; SILVA, M. K. N.; MATIAS, E. F. F. (2019). Potencial antifúngico e modulador da resistência fúngica do óleo essencial de Cordia Verbenacea Dc. exposto a irradiação de micro-ondas. Revista E-ciência, 7(1): 34-42. http://dx.doi.org/10.19095/rec.v7i1.404.

BARROSO, I. C. E.; OLIVEIRA, F. DE; BRANCO, L. H. Z.; KATO, E. T. M.; DIAS, T. G. (2002). O gênero Cordia L.: botânica, química e farmacologia. Revista Lecta, 20(1): 15-34.

BAYEUX, M. C.; FERNANDES, A. T.; FOGLIO, M. A.; CARVALHO, J. E. (2002). Evaluation of the antiedematogenic activity of artemetin isolated from Cordia curassavica DC. Brazilian Journal of Medical and Biological Research, 35: 1229-1232. http://dx.doi.org/10.1590/S0100879X2002001000017.

BERLINCK, R. G. S. (2012). Bioprospecção no Brasil: um breve histórico. Ciência e Cultura, 64(3): 2730. http://dx.doi.org/10.21800/S0009-67252012000300010.

BLANCO, M. C. S. G. (2013). Produção vegetal: Erva-baleeira. Governo do Estado de São Paulo, Secretaria de Agricultura e Abastecimento. 
BOLZANI, V. S.; VALLI, M.; PIVATTO, M.; VIEGAS JÚNIOR, C. (2012). Natural products from brazilian biodiversity as a source of new models for medicinal chemistry. Pure and Applied Chemistry, 84(9): 1837-1937. https://doi.org/10.1351/PAC-CON-12-01-11.

BRANDÃO, D. C.; BRANDÃO, G. C.; MIRANDA, J. B.; FARIA, P. A.; JESUS-GARCIA, R. (2006). Estudo fase III, duplo-cego, aleatório, comparativo para avaliar a eficácia e tolerabilidade da Cordia Verbenacea e do diclofenaco dietilamônio em pacientes portadores de contusões, entorses, traumas e lesões musculares com início inferior a 24 horas. Revista Brasileira de Medicina, 63(8): 408-415.

BRANDÃO, D. S.; COSTA, K. P.; VIEIRA, I. T. R.; FONSECA, F. S. A.; FIGUEIREDO, L. S.; MARTINS, E. R. (2017). Growing of tropical black sage and chemical composition of the essential oil. Revista Ciências Agrárias 40(4), 823-829. https://doi.org/10.19084/RCA17094.

BRANDÃO, D. S.; MENDES, A. D. R.; SANTOS, R. R.; ROCHA, S. M. G.; LEITE, G. L. D.; MARTINS, E. R. (2015). Biologia floral e sistema reprodutivo da erva-baleeira (Varronia curassavica Jacq.). Revista Brasileira de Plantas Medicinais, 17: 562-569. http://dx.doi.org/10.1590/1983$\underline{084 \mathrm{X} / 14011 .}$

BRASIL. (2006). Política Nacional de Plantas Medicinais e Fitoterápicos. Ministério da Saúde. Secretaria de Ciência, Tecnologia e Insumos Estratégicos. Departamento de Assistência Farmacêutica. Decreto no 5.813, de 22 de junho de 2006.

BRASIL. (2011). Formulário de Fitoterápicos da Farmacopéia Brasileira. Agência Nacional de Vigilância Sanitária. Brasília: Anvisa.

BRASIL. (2012). Práticas integrativas e complementares: plantas medicinais e fitoterapia na atenção básica. Ministério da Saúde. Secretaria de Atenção à Saúde. Departamento de Atenção Básica. Brasília: Ministério da Saúde, 156 p. (Série A. Normas e Manuais Técnicos) (Cadernos de Atenção Básica; n. 31).

CARVALHO JÚNIOR, P. M. DE; RODRIGUES, R. F. O.; SAWAYA, A. C. H. F.; MARQUES, M. O. M.; SHIMIZU, M. T. (2004). Chemical composition and antimicrobial activity of the oil of Cordia verbenacea DC. Journal of Ethnopharmacology, 95: 297-301. https://doi.org/10.4103/09748490.99080 .

CAYE, V. A.; SCHNEIDER, S.; FERNANDES, F. F.; SEGATTO, C.; BUBA, G. P.; LAJUS, C. R.; CERICATO, A. (2020). Estaquia de erva baleeira submetidas à fitorregulador extraído da tiririca. Revista Americana de Empreendedorismo e Inovação. v. 2, n. 1, mar.

CHRISTO, A. G.; GUEDES-BRUNI, R. R.; FONSECA-KRUEL, V. S. (2006). Uso de recursos vegetais em comunidades rurais limítrofes à Reserva Biológica de Poço das Antas, Silva Jardim, Rio de Janeiro: estudo de caso na gleba Aldeia Velha. Rodriguésia, 57(3): 519-542. https://doi.org/10.5935/2446-4775.20170013.

FACANALI, R.; MARQUES, M. O. M.; HANTAO, L. W. (2020). Metabolic Profiling of Varronia curassavica Jacq. Terpenoids by Flow Modulated Two-Dimensional Gas Chromatography $\begin{array}{lllll}\text { Coupled to Mass Spectrometry. } & \text { Separations, } & \text { 7, }\end{array}$ https://doi.org/10.3390/separations7010018. 
FACHINELLO, J. C.; HOFFMANN, A.; NACHTIGAL, J. C.; KERSTEN, E. (2005). Propagação vegetativa por estaquia. In: FACHINELLO, J. C.; HOFFMANN, A.; NACHTIGAL, J. C. (Eds). Propagação de plantas frutíferas. Brasília: Embrapa Informações Tecnológicas, p. 69-109.

FALKENBERG, M. B. (2011). Varronia Curassavica. In: CORADIN, L.; SIMINSKI, A.; REIS, A. (Eds). Espécies nativas da flora brasileira de valor econômico atual ou potencial. p. 715-719.

FEIJÓ, E. V. R. S.; OLIVEIRA, R. A.; COSTA, L. C. B. (2014). Light affects Varronia curassavica essential oil yield by increasing trichomes frequency. Revista Brasileira de Farmacognosia, 24: 516-523. https://doi.org/10.1016/j.bjp.2014.10.005.

FERNANDES, E. S.; PASSOS, G. F.; MEDEIROS, R.; CUNHA, F. M.; FERREIRA, J.; CAMPOS, M. M.; PIANOWSKI, L. F.; CALIXTO, J. B. 2007. Anti-inflammatory effects of compounds alphahumulene and (-)-transcaryophyllene isolated from the essential oil of Cordia verbenacea. European Journal of Pharmacology, 228-236. https://doi.org/10.1016/i.ejphar.2007.04.059.

FERNANDES, G. P.; KERNTOPF, M. R.; LEMOS, I. C. S. (2013). Superexploração de recursos naturais: implicações para sociobiodiversidade, a bioprospecção e a etnomedicina. Ensaios e Ciência: Ciências Biológicas, Agrárias e da Saúde, 17(2): 95-105.

FICARRA, R.; FICARRA, P.; TOMMASINI, S. (1995). Leaf extracts of some Cordia species analgesic and antiinflammatory activities as well as their chromatographic analysis. Fármaco, 50: 245256.

FIGUEIRA, G. M.; PEREIRA, B.; MAGALHÃES, P. M. (2001). Aspectos da produção de mudas de ervabaleeira Cordia curassavica (Jacq.) Roem \& Schult. V Jornada Paulista de Plantas Medicinais.

FLORA DO BRASIL. (2010). Flora do Brasil. Jardim Botânico do Rio de Janeiro. Disponível em: <http://floradobrasil.jbrj.gov.br/>. Acesso em: 23/08/2019.

FONSECA-KRUEL, V. S.; PEIXOTO, A. L. (2004). Etnobotânica na Reserva Extrativista Marinha de Arraial do Cabo, RJ, Brasil. Acta Botanica Brasilica, 18(1): 177-190. http://dx.doi.org/10.1590/S0102-33062004000100015.

FREZZA, T. F.; OLIVEIRA, A. S. S.; SIMÕES, L. F.; REHDER, V. L. G.; ALEGRETTI, S. M. (2010). Ação da fração etanólica de Cordia verbenacea na sobrevida de Schistosoma mansoni. Revista Saúde, 4(Esp. 1): p. 124.

FUNARI, C. S.; FERRO, V. O. (2005). Uso ético da biodiversidade brasileira: necessidade e oportunidade. Revista Brasileira de Farmacognosia, 15(2): 178-182. http://dx.doi.org/10.1590/S0102-695X2005000200018.

GANDOLFO, E. S.; HANAZAKI, N. (2011). Etnobotânica e urbanização: conhecimento e utilização de plantas de restinga pela comunidade nativa do distrito do Campeche (Florianópolis, SC). Acta Botanica Brasilica, v.25, p.168-177. http://dx.doi.org/10.1590/S0102-33062011000100020. 
GASPARINO, E. C.; BARROS, M. A. V. C. (2009). Palinotaxonomia das espécies de Cordiaceae (Boraginales) ocorrentes no Estado de São Paulo. Revista Brasileira de Botânica, 32: 33-55. http://dx.doi.org/10.1590/S0100-84042009000100005.

GILBERT, B; FAVORETO, R. (2012). Cordia verbenaceae DC Boraginaceae. Revista Fitos, 7(1).

GOMES, P. A.; FREITAS, S. P.; MAIA, J. T. L. S.; MARTINS, E. R. (2010). Óleo essencial da erva-baleeira (Cordia verbenacea L.) de áreas nativas. Horticultura Brasileira, 28: S3387-S3392.

HAINES-YOUNG, R. (2009). Land use and biodiversity relationships. Land Use Policy, 26(S): 178-186. https://doi.org/10.1016/j.landusepol.2009.08.009.

HARDIN, G. (1968). The tragedy of the commons. Science, 162: 1243-1248. https://doi.org/10.1126/science.162.3859.1243.

HERNÁNDEZ, T.; CANALES, M.; AVILA, J. G.; DURAN, A.; CABALLERO, J.; ROMO-DE-VIVAR, A.; LIRA, R. (2003). Ethnobotany and antibacterial activity of some plants used in tradicional medicine of Zapotitlán de Las Salinas, Puebla (México). Journal of Ethnopharmacology, 88: 181-188. https://doi.org/10.1016/S0378-8741(03)00213-7.

hOELTGebaum, M. P.; MONTAGNA, T.; LANDO, A. P.; PUTTKAMMER, C.; ORTH, A. I.; GUERRA, M. P.; REIS, M. S. (2018). Reproductive Biology of Varronia curassavica Jacq. (Boraginaceae). Anais da Academia Brasileira de Ciências, 90(1): 59-71. http://dx.doi.org/10.1590/0001$\underline{3765201820160273 .}$.

INPI. (2012). International Plant Name Index. Disponível em https://www.ipni.org/. Acesso em 23/08/2019.

$\mathrm{KOCH}, \mathrm{V}$. (2000). Estudo etnobotânico das plantas medicinais na cultura ítalo-brasileira no Rio Grande Do Sul. Um modelo para o cultivo comercial na agricultura familiar. Dissertação de mestrado (UNISINOS): Porto Alegre, RS.

LANS, C.; HARPER, T.; GEORG E.; K.; BRIDGEWATER, E. (2000). Medicinal plants used for dogs in Trinidad and Tobago. Preventive Veterinary Medicine, 45: 201-220. https://doi.org/10.1016/S0167-5877(00)00123-9.

LEAL-COSTA, M. V.; AMÉLIA, R. P. (2017). Anatomia foliar de Varronia curassavica Jacq. (Cordiaceae). Revista Fitos, Rio de Janeiro, Vol. 11(1), 1-118. https://doi.org/10.5935/24464775.20170004.

LEONEL, M. (2000). Bio-sociodiversidade: preservação e mercado. Estudos Avançados, 14(38): 321 346. http://dx.doi.org/10.1590/S0103-40142000000100019.

LORENZI, H.; MATOS, F. J. A. (2008). Plantas medicinais no Brasil: nativas e exóticas cultivadas. Nova Odessa: Instituto Plantarum. 544 p.

LOURENZANI, A. E. B. S. (2004). Barreiras e oportunidades na comercialização de plantas medicinais provenientes da agricultura familiar. Informações Econômicas, 34: 15-25. 
MACHADO, T. M.; JANANI, J. K.; FERREIRA, M. I.; ONO, E. O.; LIMA, G. P. P. (2011). Comportamento fisiológico de sementes de Cordia verbenacea DC (Boraginaceae) sob diferentes substratos, temperaturas e luz. Naturalia, 34: 86-95.

MACIEL, M. A. M.; PINTO, A. C.; VEIGA Jr., V. F. (2002). Plantas medicinais: a necessidade de estudos multidisciplinares. Química Nova, 25(3): 429-438.

MAIOLI-AZEVEDO, V.; FONSECA-KRUEL, V. S. (2007). Plantas medicinais e ritualísticas vendidas em feiras livres no município do Rio de Janeiro, RJ, Brasil: estudo de caso nas Zonas Norte e Sul. Acta Botanica Brasilica, 21(2): 263-275. http://dx.doi.org/10.1590/S010233062007000200002.

MARQUES, M. C. M.; OLIVEIRA, P. E. A. M. (2005). Características reprodutivas das espécies vegetais da planície litorânea. In: MARQUES, M. C. M; BRITEZ, R. M. (Org.). História natural e conservação da Ilha do Mel. Curitiba: UFP. p.85-101.

MATIAS, E. F. F.; SANTOS, K. K. A.; ALMEIDA, T. S.; COSTA, T. G. M.; HENRIQUE, H. D. M. (2010). Enhancement of antibiotic activity by Cordia verbenacea DC. Latin American Journal of Pharmacy, 29(6): 1049-52.

MATIAS, E. F. F.; BEZERRA, V. B.; BOMFIM, R. O.; LUCENA, A. L. V. M.; LEITE, V. D.; FEITOSA, J. H. F.; SILVA, M. K. N. (2017). Avaliação da atividade antibacteriana e moduladora do óleo essencial de Cordia verbenacea DC. associado às luzes de led. Revista Interfaces, 5(14): 07-14. https://doi.org/10.16891/2317-434X.v5.e14.a2017.pp07-14.

MECCIA, G.; ROJAS, L. B.; VELASCO, J.; DÍAZ, T.; USUBILLAGA, A.; ARZOLA, J. C.; RAMOS, S. (2009). Chemical composition and antibacterial activity of the essential oil of Cordia verbenacea from the Venezuelan Andes. Natural Product Communications, 4(8).

MEDEIROS, R.; PASSOS, G. F.; VITOR, C. E.; KOEPP, J., MAZZUCO, T. L.; PIANOWSKI, L. F.; CAMPOS, M. M.; CALIXTO, J. B. (2007). Effect of two active compounds obtained from the essential oil of Cordia Verbenacea on the acute inflammatory responses elicited by Ips in the rat paw. British Journal Of Pharmacology, 151(5), 618-627. https://doi.org/10.1038/sj.bjp.0707270.

MELO, J. I. M.; SALES, M. F. (2005). Boraginaceae A. Juss. na região de Xingó: Alagoas e Sergipe. Hoehnea, 32(3): p.369-380.

MELO, S.; LACERDA, V. D.; HANAZAKI, N. (2008). Espécies de restinga conhecidas pela comunidade do Pântano do Sul, Florianópolis, Santa Catarina, Brasil. Rodriguésia, 59(4): 799-812. http://dx.doi.org/10.1590/2175-7860200859409.

MENDES, A. D. R.; LACERDA, T. H. S.; ROCHA, S. M. G.; MARTINS, E. R. (2014). Reguladores vegetais e substratos no enraizamento de estacas de erva-baleeira (Varronia curassavica Jacq.) Revista Brasileira de Plantas Medicinais, 16(2): 262-270. http://dx.doi.org/10.1590/S1516$\underline{05722014000200015}$.

MICHIELIN, E. M. Z.; SALVADOR, A. A.; RIEHL, C. A. S.; SMÂNIA JUNIOR, A.; SMÂNIA, E. F. A.; FERREIRA, S. R. S. (2009). Chemical composition and antibacterial activity of Cordia verbenacea 
extracts. Bioresource Technology, 100: 6615-6623. https://doi.org/10.4103/09748490.99080 .

MILLER, J. S.; GOTTSCHLING, M. (2007). Generic classification in the Cordiaceae (Boraginales): resurrection of the genus varronia. P. Br. Taxon, 56(1): 163-169. https://doi.org/10.2307/25065747.

MIRANDA, T. M.; HANAZAKI, N. (2008). Conhecimento e uso de recursos vegetais de restinga por comunidades das Ilhas do Cardoso (SP) e de Santa Catarina (SC), Brasil. Acta Botanica Brasilica, 22(1): 203-215. http://dx.doi.org/10.1590/S0102-33062008000100020.

MONTANARI JUNIOR, I (2011). Variabilidade genética em uma população de Cordia verbenacea DC. para características agronômicas e fitoquímicas. Tese de Doutorado (Universidade Estadual Paulista, Faculdade de Ciências Agronômicas), Botucatu.

MONTANARI JUNIOR, I. (2000). Aspectos do cultivo comercial de erva baleeira. Revista Agroecologia Hoje, 3(2): 14-15.

MORANDI, M. A. B. (2009). Integração de métodos físicos e biológicos no controle de doenças em viveiros de plantas medicinais: estudo de caso com Cordia verbenacea. IN: BETTIOL, W.; MORANDI, M. A. B. (Ed.). Biocontrole de Doenças de Plantas: Uso e Perspectivas. Jaguariúna: EMBRAPA Meio Ambiente. p.337-341.

MOURA, D. C.; MELO, J. I. M.; SCHLINDWEIN, C. (2007). Visitantes Florais de Boraginaceae A. Juss. no baixo curso do Rio São Francisco: Alagoas e Sergipe. Revista Brasileira de Biociências, 5(supl.1): 285-287.

NASCIMENTO, A. S.; MESQUITA, A. L. M.; CALDAS, E. R. C. (1986). Flutuação populacional e Manejo da broca da laranjeira, Cratosomus flavofasciatus Guerrin (Coleoptera: Curculionidae) com "maria preta" Cordia verbenaceae (Borraginaceae). Anais da Sociedade Entomológica do Brasil, 15(Supl): 125-134.

OLER, J. R. L.; AMOROZO, M. C. M.; MONTEIRO, R.; BUTTURI-GOMES, D. (2019). Etnobotânica de plantas tóxicas como subsídio para campanhas de prevenção de acidentes: um estudo de caso em Cananéia, São Paulo, Brasil. Scientia Plena, 15(11). https://doi.org/0.14808/sci.plena.2019.111201.

OLIVEIRA Jr., C. J. F.; CABREIRA, P. P. (2012). Sistemas agroflorestais: potencial econômico da biodiversidade a partir do conhecimento tradicional ou local. Revista Verde de Agroecologia e Desenvolvimento Sustentável, 7(1): 212-224.

OLIVEIRA JÚNIOR, C. J. F.; CABREIRA, P. P.; BEGOSSI, A. (2012). The dilemma of plant knowledge and compensation for native people living in brazilian biomes. Journal of Ecosystem \& Ecography, 2(2): 100108. https://doi.org/10.4172/2157-7625.1000108.

OLIVEIRA JÚNIOR, C. J. F.; SANTOS, J. L.; MÁXIMO, H. C. (2014). Agroecology and environmental services. Nature and Conservation, 7(1): 19-32. https://doi.org/10.6008/SPC23182881.2014.001.0002. 
OLIVEIRA JÚNIOR, C. J. F.; VOIGTEL, S. D. S.; NICOLAU, S. A.; ARAGAKI, S. (2018). Sociobiodiversidade e agricultura familiar em Joanópolis/SP, Brasil: potencial econômico da flora local. Hoehnea, 45(1): 40-54. http://dx.doi.org/10.1590/2236-8906-78/2017.

OSHADHI. (2018). Black sage, Cordia verbenacea DC. Disponível em http://www.oshadhi.co.uk/black-sage-cordia-verbenacea-d-c/ (acesso em 16-10-2018).

PARISOTTO, E. B.; MICHIELIN, E. M. Z.; BISCARO, F.; FERREIRA, S. R. S.; FILHO D. W.; PEDROSA, R. C. (2012). The antitumor activity of extracts from Cordia verbenacea D.C. obtained by supercritical fluid extraction. The Journal of Supercritical Fluids, 61: 101-107. https://doi.org/10.1016/j.supflu.2011.08.016.

PASSOS, G. F.; FERNANDES, E. S.; CUNHA, F. M.; FERREIRA, J.; PIANOWSKI, L. F.; CAMPOS, M. M.; CALIXTO, J. B. (2007). Anti-inflammatory and anti-allergic properties of the essential oil and active compounds from Cordia verbenacea. Journal of Ethnopharmacology, 110(2): 323-333. https://doi.org/10.1016/i.jep.2006.09.032.

PEREIRA, A. V. G.; ALBIERO, A. L. M. (2015). A valorização da utilização de plantas medicinais na atenção básica: oficinas de aprendizagem. Arquivos do Mudi, 19(2-3): 23-42. https://doi.org/10.4025/arqmudi.v19i2-3.30055.

PIANOWSKI, L. (2005). Primeiro fitomedicamento baseado em planta brasileira é um antiinflamatório. Jornal Phytomédica, 1(1): 2-3.

PINHO, L.; SOUZA, P. N. S.; SOBRINHO, E. M.; ALMEIDA, A. C.; MARTINS, E. R. (2012). Atividade antimicrobiana de extratos hidro alcóolicos das folhas de alecrim-pimenta, aroeira, barbatimão, erva-baleeira e do farelo da casca de pequi. Ciência Rural, 42(2): 326-331. http://dx.doi.org/10.1590/S0103-84782012005000003.

PINTO, E. P. P.; AMOROZO, M. C. M.; FURLAN, A. (2006). Conhecimento popular sobre plantas medicinais em comunidades rurais de Mata Atlântica - Itacaré, BA, Brasil. Acta Botanica Brasilica, 20(4): 751-762. http://dx.doi.org/10.1590/S0102-33062006000400001.

QUEIROZ, T. B.; FONSECA, F. S. A.; MENDES, A. D. R.; AZEVEDO, A. M.; MARTINS, E. R. (2020). Chemical diversity of accessions of the in vivo germplasm bank of Varronia curassavica (Jacq.). Acta Scientiarum Agronomy, 42: e42725. https://doi.org/10.4025/actasciagron.v42i1.42726.

QUISPE-CONDORI, S. (2008). Obtaining $\beta$-caryophyllene from Cordia verbenacea de Candolle by supercritical fluid extraction. Journal of Supercritical Fluids, 46: 27-32. https://doi.org/10.1016/i.supflu.2008.02.015.

RIBEIRO, D. A.; MACEDO, D. G.; OLIVEIRA, L. G. S.; SANTOS, M. O.; ALMEIDA, B. V.; MACEDO, J. G. F.; MACEDO, M. J. F.; SOUZA, R. K. D.; ARAÚJO, T. M. S.; SOUZA, M. M. A. (2019). Conservation priorities for medicinal woody species in a Cerrado area in the Chapada do Araripe, northeastern Brazil. Environment Development Sustainable, 21: 61-77. https://doi.org/10.1007/s10668-017-0023-9.

RIBEIRO, P. G. F.; DINIZ, R. C. (2008). Cultivo de plantas aromáticas e medicinais. Instituto Agronômico do Paraná. 218 p. 
RODAL, M. J. R.; NASCIMENTO, L. M. (2002). Levantamento florístico da floresta serrana da Reserva Biológica de Serra Negra, microrregião de Itaparica, Pernambuco, Brasil. Acta Botanica Brasilica, 16(4): 481-500. http://dx.doi.org/10.1590/S0102-33062002000400009.

RODRIGUES, A. C. C.; GUEDES, M. L. S. (2006). Utilização de plantas medicinais no Povoado Sapucaia, Cruz das Almas - Bahia. Revista Brasileira de Plantas Medicinais, 8(2), 1-7.

RODRIGUES, A. K. C.; PAIVA, I. C.; BORSATO, A. V. (2011). Rendimento de óleo essencial em folhas desidratadas e frescas de Cordia verbenaceae DC. Cadernos de Agroecologia, 6(2).

RODRIGUES, A. K. C.; BORSATO, A. V.; JORGE, M. H. A.; BISPO, W.; DURAN, F. S.; ARRUDA, K. C. R. (2010). Enraizamento de estacas de Cordia Verbenacea Dc. tratadas com Cyperus Rotundus L. Cadernos de Agroecologia, 5(1).

RODRIGUES, F. F. G.; OLIVEIRA, L. G. S.; RODRIGUES, F. F. G.; SARAIVA, M. E.; ALMEIDA, S. C. X.; CABRAL, M. E. S.; CAMPOS, A. R.; COSTA, J. G. M. (2012). Composição química, atividade antibacteriana e antifúngica do óleo essencial de Cordia verbenacea DC. Pharmacognosy, 4(3): 161-165.

ROLDÃO, E. F.; WITAICENIS, A.; SEITO, L. N.; HIRUMA-LIMA, C. A.; DI STASI, L. C. (2008). Evaluation of the antiulcerogenic analgesic activities of Cordia Verbenacea DC. (Boraginaceae). Journal Of Ethnopharmacology, 119(1-2): 94-98. https://doi.org/10.1016/j.jep.2008.06.001.

ROSA, D. D.; BASSETO, M. A.; FELICIANO, F.; NEVES, M. B.; BALDIN, E. L. L. (2008). Ocorrência de Dictyla monotropidia Stål (Hemiptera: Tingidae) em Cordia verbenacea Al. DC no Brasil. Neotropical Entomology, 37(2): 236-238. http://dx.doi.org/10.1590/S1519566X2008000200021.

SÁNCHEZ, J. E. (1995). Cordia subgénero Varronia (Boraginaceae). Flora de Colombia, 14: 1-176.

SANTONIERI, L.; BUSTAMONTE, P.G. (2016). Conservação ex situ e on farm de recursos genéticos: desafios para promover sinergias e complementaridades. Boletim Museu Paraense Emílio Goeldi (Ciências Humanas), 11(3): 677-690. http://dx.doi.org/10.1590/1981.81222016000300008.

SANTOS, A. S.; REHDER, V. L. G.; PIANOWSKI, L. F.; CALIXTO, J. B.; MAGALHÃES, P. M. (2007). Composição química de frações ativas do óleo essencial de Cordia verbenacea DC. 30a Reunião Anual da Sociedade Brasileira de Química, Águas de Lindóia - SP.

SANTOS, R. P.; NUNES, E. P.; NASCIMENTO, R. F.; SANTIAGO, G. M. P.; MENEZES, G.H. A.; SILVEIRA, E. R.; PESSOA, O. D. L. (2006). Chemical composition and larvicidal activity of the essential oils of Cordia leucomalloides and Cordia curassavica from the Northeast of Brazil. Journal of the Brazilian Chemical Society, 17: 1027-1030. http://dx.doi.org/10.1590/S0103$\underline{50532006000500030 .}$.

SANTOS, S. C; MELLO, J. C. P. (2004). Taninos. In: SIMÕES, C. M. O.; SCHENKEL, E. P.; GOSMANN, G.; MELLO, J. C. P.; MENTZ, L. A.; PETROVICK, P. R. (Eds.). Farmacognosia: da planta ao medicamento. p.527-554. 
SCARDELATO, J. A.; SANTOS, A. G.; PIETRO, R. C. L. R.; SACRAMENTO, L. V. S. (2016). Cordia verbenacea DC. (Boraginaceae): quality control parameters, phytochemical screening and evaluation of anti-candida activity. Revista Brasileia de Plantas Medicinais, 18: 837-843.

SCIARRONE, D.; GIUFFRIDA, D.; ROTONDO, A.; MICALIZZI, G.; ZOCCALI, M.; PANTÔ, S.; DONATO, P.; DORES, R. G. R.; MONDELLO, L. (2017). Quali-quantitative characterization of the volatile constituents in Cordia verbenacea D.C. essential oil exploiting advanced chromatografhic approaches and nuclear magnetic resonance analysis. Journal of Chromatography, 1524: 246253. https://doi.org/10.1016/i.chroma.2017.10.007.

SERTIÉ, J. A. A.; BASILE, A. C.; PANIZZA, S.; MATILDA, A. K.; ZELNIK, R. (1988). Pharmacological assay of Cordia verbenacea; Part 1 anti-Inflammatory activity and toxicity of the crude extract of the leaves. Planta Med, 54(1): 7-10. https://doi.org/10.1055/s-2006-962318.

SERTIÉ, J. A. A.; BASILE, A. C.; PANIZZA, S.; OSHIRO, T. T.; AZZOLINI, C. P.; PENNA, S. C. (1991). Pharmacological assay of Cordia verbenacea III: oral and topical antiinflamatory activity and gastrotoxicity of a crude leaf extract. Journal of Ethnopharmacology, 31(2): 239-247. https://doi.org/10.1016/0378-8741(91)90008-2.

SERTIÉ, J. A.; WOISKY, R. G.; WIEZEL, G.; RODRIGUES, M. (2005).Pharmacological assay of Cordia verbenacea $\mathrm{V}$ : oral and topical anti-inflammatory activity, analgesic effect and fetus toxicity of a crude leaf extract. $\quad$ Phytomedicine,12(5): 338-44. https://doi.org/10.1016/i.phymed.2003.09.013.

SILVA JÚNIOR, A. A.; VIZZOTO, V. J.; GIORGI, E. (1995). Plantas medicinais caracterização e cultivo. Florianópolis: EPAGRI. $71 \mathrm{p}$.

SMITH, L. B. (1970). Boragináceas. In: R. REITZ (ed.). Flora Ilustrada Catarinense. Itajaí: Herbário Barbosa Rodrigues. 85p.

SOUZA, M. R. M.; PEREIRA, R. G. F.; FONSECA, M. C. M. (2012). Comercialização de plantas medicinais no contexto da cadeia produtiva em Minas Gerais. Revista Brasileira de Plantas Medicinais, 14(esp): 242-245. http://dx.doi.org/10.1590/S1516-05722012000500019.

THE PLANT LIST. (2013). The Plant List. Version 1.1. Disponível em: <http://www.theplantlist.org/>. Acesso em: 21/08/2019.

TICLI, F. K.; HAGE, L. I. S.; CAMBRAIA, R. S.; PEREIRA, P. S.; MAGRO, A. J.; FONTES, M. R. M.; STÁBELI, R. G.; GIGLIO, J. R.; FRANÇA, S. C.; SOARES, A. M.; SAMPAIO, S. V. (2005). Rosmarinic acid, a new snake venom phospholipase A2 inhibitor from Cordia verbenacea (Boraginaceae): antiserum action potentation and molecular interaction. Toxicon, 46: 318-327. https://doi.org/10.1016/j.toxicon.2005.04.023.

VENTRELLA, M. C.; MARINHO, C. R. (2008). Morphology and histochemistry of glandular trichomes of Cordia verbenacea DC. (Boraginaceae) leaves. Revista Brasileira de Botânica, 31(3): 457-467. http://dx.doi.org/10.1590/S0100-84042008000300010.

YUNES, R. A.; CALIXTO J. B. (2001). Plantas medicinais sob a ótica da química moderna. Chapecó: Agros. 500p. 